\title{
A massively parallel multiscale CAFE framework for the modelling of fracture in heterogeneous materials under dynamic loading \\ DOI:
}

10.1016/j.advengsoft.2019.102737

\section{Document Version \\ Accepted author manuscript}

Link to publication record in Manchester Research Explorer

Citation for published version (APA):

Hewitt, S., Margetts, L., Shterenlikht, A., \& Revell, A. (2020). A massively parallel multiscale CAFE framework for the modelling of fracture in heterogeneous materials under dynamic loading. Advances in Engineering Software. https://doi.org/10.1016/j.advengsoft.2019.102737

\section{Published in:}

Advances in Engineering Software

\section{Citing this paper}

Please note that where the full-text provided on Manchester Research Explorer is the Author Accepted Manuscript or Proof version this may differ from the final Published version. If citing, it is advised that you check and use the publisher's definitive version.

\section{General rights}

Copyright and moral rights for the publications made accessible in the Research Explorer are retained by the authors and/or other copyright owners and it is a condition of accessing publications that users recognise and abide by the legal requirements associated with these rights.

\section{Takedown policy}

If you believe that this document breaches copyright please refer to the University of Manchester's Takedown Procedures [http://man.ac.uk/04Y6Bo] or contact uml.scholarlycommunications@manchester.ac.uk providing relevant details, so we can investigate your claim.

\section{OPEN ACCESS}




\title{
A massively parallel multiscale CAFE framework for the modelling of fracture in heterogeneous materials under dynamic loading
}

\author{
Sam Hewitt ${ }^{\mathrm{a}}$, Lee Margetts $^{\mathrm{a}, *}$, Anton Shterenlikht $^{\mathrm{b}}$, Alistair Revell $^{\mathrm{a}}$ \\ ${ }^{a}$ School of Mechanical, Aerospace and Civil Engineering, The University of Manchester, Manchester, M13 9PL \\ ${ }^{b}$ Faculty of Engineering, University of Bristol, Bristol, BS8 1 TH
}

\begin{abstract}
This paper presents a novel computational framework for modelling multiscale fracture that can be used to solve engineering problems subject to dynamic loading. The framework simulates, mechanistically, at the mesoscale, the physical processes that lead to brittle fracture. A homogenisation step is used to translate the accumulation of damage from the mesoscale to the macroscale (as a reduced stiffness in the corresponding region of the structure). In order to achieve this, the multiscale framework couples together two open source Fortran packages; the macroscale ParaFEM with the mesoscale CASUP. ParaFEM is a highly parallel finite element analysis library used to model structures at the continuum scale. CASUP is a package that uses cellular automata to simulate brittle fracture in polycrystalline materials. A simple test problem involving a vibrating cantilever beam is used to demonstrate the simulation of dynamic cyclic loading, leading to brittle cracking. In the cellular automata software, there are a range of parameters that can be adjusted, such as the fracture energy and grain size. These are explored to demonstrate how they might affect the predicted structural integrity of the cantilever beam. Parallel performance is investigated using a Cray XC30 supercomputer, showing that the software can make efficient use of tens of thousands of cores. This paper highlights that modelling the physical mechanisms that lead to damage and plasticity could be an attractive alternative to phenomenological constitutive models. This work will be of interest to researchers and practitioners needing more precise predictions or a better understanding of damage propagation under cyclic or impact loading. With further development, this type of framework will enable the insilico design and evaluation of new material microstructures; leading to improved performance of components and devices subject to extreme operating conditions.
\end{abstract}

Keywords: Multiscale; Parallel; Fracture; Cellular Automata; Finite Element.

\section{Introduction}

Accurately capturing the physics of structures at mul- ${ }^{21}$ tiple scales offers a difficult challenge, both numerically ${ }^{22}$ and computationally. Multiscale models often involve the ${ }^{23}$ solution to physical phenomena that cannot be modelled ${ }^{24}$ using the same methodologies. Traditional continuum ${ }^{25}$ methods homogenizing the material properties at engi- ${ }^{26}$ neering length scales, cannot provide the same level of ac- ${ }^{27}$ curacy and fidelity when applied to modelling the physics ${ }^{28}$ at the mesoscale ( $\mathrm{mm}$ to $\mu \mathrm{m}$ ). The coupling of different ${ }^{29}$ methodologies, to solve each problem and capture the ${ }^{30}$ various scales and physics, offers a promising solution to ${ }^{31}$ the problem.

The highly heterogeneous nature of fracture and dis- ${ }^{33}$ continuities in brittle materials makes modelling these ${ }^{34}$ phenomena difficult. There are a number of methods in ${ }^{35}$ the literature used to capture the fracture and mesoscale ${ }^{36}$ physics in structures. Cohesive zone models (CZM) intro- ${ }^{37}$ duced in the late sixties by Dugdale [1] and Barenblatt [2], ${ }_{39}^{38}$

\footnotetext{
* Corresponding author

Email address: lee.margetts@manchester.ac.uk (Lee Margetts)
}

20

21

\author{
tion
}

24 part

$\mathrm{t}$ els, the crack fronts follow the inter element contours and so they can be computationally expensive, as high levels of refinement are required around crack fronts to obtain mesh independent results. CZM can be treated extrinsically or intrinsically based on the cohesive zones assumed pre-fracture response, with both methods having numerical challenges [6]. One of the requirements for fracture models is to simulate complex three-dimensional fracture, that can be computationally expensive. In general intrinsic approaches are often more easily parrallelised, however researchers have developed hybrid approaches [7] that maintain the parallel scalability whilst avoiding wave propagation issues common amongst intrinsic methods.

To combat these pitfalls, extended finite element methods (X-FEM) were developed [8]. These discrete models differ from the constitutive models used in CZM, using local enrichment functions within the elements to capture

August 14, 2019 
discontinuities. They have been used to model fracture ${ }_{100}$ that does not follow the path of inter element surfaces [9].101 $\mathrm{X}$-FEM methods require some input via the user, such as102 crack propagation directions [8] or maximum energy re-103 lease in crack extension [10]. Researchers have developed ${ }_{104}$ coupled XFEM/CZM models to preserve the advantages105 of both approaches [11]. In general, X-FEM methods106 have been shown to provide good approximations of frac- 107 ture, however when applied to multiscale applications the 108 model requires significantly different levels of refinement ${ }_{109}$ to capture each of the length scales [12].

Finally and more recently, the use of multiphase field ${ }_{111}$ models have been used to study anisotropic crack prop-112 agation in polycrystalline materials $[13,14,15]$. The 113 modelling approach, based on the work by Francfort and ${ }_{114}$ Marigo [16] in 1998, uses an added variable known as 115 the phase field variable to smear the crack, reducing the ${ }_{116}$ numerical difficulties of dealing with discontinuities. Fur-117 thermore, mean-field homogenization methods have been 118 used to model damage in carbon-fiber reinforced composites [17].

In comparison to these approaches that broadly char- ${ }^{119}$ acterize the material as a continuum, methods exist in $_{120}$ which the microstructure is modelled as a separate do- ${ }_{121}$ main and embedded within the larger model explicitly. ${ }_{122}$ In these models each domain captures the physics at its ${ }_{123}$ length scale and the information is passed from domain to ${ }_{124}$ domain through various boundary conditions and damage parameters. The level of coupling of multiscale mod- ${ }_{125}$ els is categorised into hierarchical [18] in which detail is only passed from the fine scale to the coarse scale, semi- ${ }^{126}$ concurrent in which information is passed to either scale ${ }^{127}$ [19] and concurrent in which both scales are solved simul- ${ }^{128}$ taneously and compatibility conditions are used to couple ${ }^{129}$ the two [20].

Within these categorisations, researchers have used $\mathrm{a}^{13}$ range of approaches, including multi level FEM $\left(\mathrm{FE}^{2}\right)^{132}$ [21], coupled FEM to molecular dynamics (MD) [22], us- ${ }^{133}$ ing FEM and X-FEM to model different scales within ${ }^{134}$ the structure [23] and coupled cellular-automata $(\mathrm{CA})^{135}$ and FEM methods [24]. CAFE models have been used ${ }^{13}$ to model a range of phenomena, including solidification [25] and recrystallisation [26] and more recently to model ${ }^{137}$ damage development and cleavage in quasi brittle mate- ${ }^{138}$ rials [27, 28]. A summary of multiscale strategies used ${ }^{139}$ for solving fracture problems is provided in Table 1.

In 2015 Shterenlikht and Margetts [24] released a mas- ${ }^{141}$ sively parallel CAFE framework for modelling brittle fracture in polycrystalline materials, coupling a general cellular automata library, CASUP, with a highly parallel finite ${ }_{142}$ element package ParaFEM. The paper dealt with the in- ${ }_{143}$ cremental loading of a static equilibrium problem, exclud- ${ }_{144}$ ing any dynamic effects. The model falls into the cate- ${ }_{145}$ gory of semi-concurrent multiscale models passing stress ${ }_{146}$ fields from the macroscale to the mesoscale and damage parameters back the other way. A key advantage of the framework is its highly parallel implementation. In
2018 Shterenlikht et al [34] highlighted the applicability of coarray Fortran to the CA framework and the scalability it could achieve. This paper describes a new mini-app that uses the framework described in the original article with the CAFE model being adapted to simulate transient, dynamically loaded problems. The paper presents completely novel results, detailing the applications scalability and providing an example test case analysing both the mesoscale and macroscale results.

The paper is structured as follows: Section 2 describes the numerical and mechanistic methodologies of ParaFEM and CASUP respectively, along with an overview of the parallel techniques of both the packages. Section 3 provides the numerical results to a test problem looking at the deformation of a cantilever beam that undergoes brittle fracture. Section 4 gives a detailed breakdown of the time expense and scalability of the application. Finally, Section 5 provides a summary and discussion about the results achieved.

\section{Method}

The applications are coupled in a segregated manner with it being possible to run each package independently. It is therefore suitable to highlight the numerical methodology and parallel implementation of each of the packages independently.

\subsection{Engineering Scale : ParaFEM}

The continuum scale of the problem is modelled numerically using FEM. ParaFEM is a FEM toolbox consisting of a series of libraries and mini-apps. The toolbox written in Fortran was specifically developed to be highly parallel and run on a variety of large high performance computing facilities [35]. Each mini-app [36] solves a specific engineering problem, some examples include: heat transfer [37], stochastic finite element modelling [38], geometric nonlinearity [39], material nonlinearity [40], image-based modelling [41] and fluid structure interaction [42].

\subsubsection{Numerical Model}

The governing equations describing the dynamic equilibrium of a structure, rely on the conservation of momentum. The strong form of the equation for the linear momentum balance is shown in Equation 1.

$$
\rho \ddot{\mathbf{u}}=\nabla \cdot \boldsymbol{\sigma}+\rho \mathbf{b},
$$

where $\boldsymbol{\sigma}$ is the Cauchy stress tensor, $\ddot{\mathbf{u}}$ is the second time derivative of the displacement $\mathbf{u}, \rho$ the solid density and b the body force per unit mass. Multiplying the strong form of the equation by the virtual displacement, $\delta \mathbf{u}$, integrating over the domain, $\Omega$ and applying Gauss's diver- 


\begin{tabular}{ccc}
\hline \hline Method & Description & Papers \\
\hline Continuum/Continuum & $\begin{array}{c}\text { Coupled continum models such as finite element to finite } \\
\text { element simulations each modelling a different scale }\end{array}$ & {$[29,30,19]$} \\
Atomistic/Continuum & Molecular dynamics and discrete elements methods are & {$[31,32,33]$} \\
Mechanistic/Continuum & $\begin{array}{c}\text { coupled to finite element models } \\
\text { Mechanistic models such as cellular automata are coupled } \\
\text { to finite element models }\end{array}$ & {$[24,34,27]$} \\
\hline \hline
\end{tabular}

Table 1: Summary of general multiscale strategies to solving damage and fracture problems, with example papers.

gence theorem, results in the expression of virtual work,176 Equation 2.

$$
\begin{gathered}
\int_{\Omega} \rho \delta \mathbf{u}^{T} \ddot{\mathbf{u}} d \Omega \\
+\int_{\Omega} \hat{\varepsilon}^{T} \boldsymbol{\sigma} d \Omega-\int_{\Gamma} \delta \mathbf{u}^{T} \mathbf{t} d \Gamma \\
-\int_{\Omega} \rho \delta \mathbf{u}^{T} \mathbf{b} d \Omega=0 .
\end{gathered}
$$

In the FEM the arbitrary body is represented by an ${ }^{179}$ aggregate of finite elements, linked though their nodes ${ }^{180}$ and edges. The displacement in each element is described ${ }^{18}$ locally through the element shape functions, $\mathbf{u}=\mathbf{N} \tilde{\mathbf{u}}$. where $\mathbf{N}$ are the element shape functions and $\tilde{\mathbf{u}}$ the local ${ }^{182}$ nodal displacements. The relationship between the ele- ${ }^{183}$ mental strains and displacements is given by, $\varepsilon=\mathbf{B u}{ }^{184}$ where $\mathbf{B}$, is the strain-displacement matrix. The fourth ${ }^{185}$ order tensor of elastic modulus, $\mathbb{C}$ is used to couple the ${ }^{186}$ elemental strains to stresses, $\sigma=\mathbb{C} \varepsilon$. Using the described ${ }^{187}$ relationships, the corresponding virtual work expression ${ }^{188}$ in its $\mathrm{FE}$ form is provided, Equation 3,

$$
\begin{aligned}
\int_{\Omega} \rho \mathbf{N}^{T} \mathbf{N} \ddot{\tilde{u}} d \Omega & +\int_{\Omega} \mathbf{B}^{T} \mathbb{C} \mathbf{B} \tilde{\mathbf{u}} d \Omega-\int_{\Gamma} \mathbf{N}^{T} \mathbf{t} d \Gamma \\
& -\int_{\Omega} \rho \mathbf{N}^{T} \mathbf{b} d \Omega=0
\end{aligned}
$$

196

where each expression in the equation can be expressed197 as a matrix. The mass matrix $\mathbf{M}=\int_{\Omega} \rho \mathbf{N}^{T} \mathbf{N} d \Omega$, the198 stiffness matrix $\mathbf{K}=\int_{\Omega} \mathbf{B}^{T} \mathbf{C B} d \Omega$ and the external forces ${ }^{199}$ on the structure $\mathbf{f}_{e x t}=\int_{\Gamma} \mathbf{N}^{T} \mathbf{t} d \Gamma-\int_{\Omega} \rho \mathbf{N}^{T} \mathbf{b} d \Omega$. This ${ }^{200}$ reduces Equation 3 to Equation 4.

$$
\mathbf{M} \ddot{\tilde{\mathbf{u}}}+\mathbf{K} \tilde{\mathbf{u}}=\mathbf{f}_{e x t}
$$

At the macroscale, the relationship between the stress $_{205}$ and strain, the constitutive law, determines the solution ${ }_{206}$ to the macroscale problem. The application presented $\mathrm{in}_{207}$ this article uses a small strain assumption and the ma- ${ }_{208}$ terial is determined to be isotropic, linear, and elastic. ${ }_{209}$ Although, it will be noted later, damage accumulated at the mesoscale results in a reduced stiffness for relevant $\mathrm{fi}_{-210}$ nite elements, the macroscale analysis is always for small strain elasticity. The transient problem is stepped in time ${ }_{212}^{21}$ utilising a linear relationship between the solution at time $\mathrm{n}$ and the solution at time $\mathrm{n}+1$. The following assumptions are made within the scheme:

$$
\begin{aligned}
& \mathbf{u}_{n+1}=\mathbf{u}_{n}+\Delta t\left[(1-\theta) \dot{\mathbf{u}}_{n}+\theta \dot{\mathbf{u}}_{n+1}\right] \\
& \dot{\mathbf{u}}_{n+1}=\dot{\mathbf{u}}_{n}+\Delta t\left[(1-\theta) \ddot{\mathbf{u}}_{n}+\theta \ddot{\mathbf{u}}_{n+1}\right]
\end{aligned}
$$

The variable $\theta$ varies between $\frac{1}{2}$ and 1 , with $\theta=\frac{1}{2}$ being equivalent of Newmarks $\beta=0.25$ method [43], with average acceleration. The results presented in this work all use a $\theta=\frac{1}{2}$, which is second order accurate in time.

\subsubsection{Parallel Implementation}

ParaFEM makes use of the message passing interface (MPI) for its parallel communications. The strategy uses an element-by-element technique that spreads both the memory and computational requirements across all the cpus. The problem is distributed evenly across the cpus with each cpu holding only its local information, this avoids building any global matrices that can be memory intensive and time consuming. The preconditioned conjugate gradient (PCG) method is utilised to iteratively solve the system of equations for the FE problem. A ParaFEM mpi module is used to hide the low level calls to the MPI routines. This module contains a number of routines that are used to gather and scatter data according to a map that highlights nodes existing on the different cpus. ParaFEM driver programs using this method have shown good scaling on 32,000 cores [44]. Finally, the I/O of ParaFEM involves the master processor reading the data from file and distributing it to each of the processors. This method although can present a bottleneck avoids the writing of a mesh file for each processor that may cause file limit issues as codes attempt to begin using petascale and exascale systems. There is an alternative to this write method. MPI-IO is an extension to MPI and offers capabilities for concurrent reading and writing to a single file from multiple processors. Libraries, such as HDF5, have utilised this extension offering very fast parallel write capabilities.

\subsection{Mesoscale : CASUP}

The previous section described the model at the engineering scale, the following section will elucidate the methodology used to capture the fracture within a heterogeneous material. Simulating fracture at the mesoscale 
can provide an accurate prediction of material strength 250 and its estimated failure point. Polycrystalline materials251 are composed of many crystals or grains that vary in size and orientation. The composition of these materials at the crystal scale has a significant impact on the material at the macro scale. The highly heterogeneous properties of these crystals can be captured and modelled using CASUP, and CA. The CA is a complete digital framework, being discrete in space, time and state. A uniform grid of cells is each assigned a state. Each step the cells state is updated using a series of simple rules. These simple rules often lead to complex physical phenomena at a more global scale within the CA grid. The following subsection will describe how the CA is used to firstly represent a heterogeneous polycrystalline material and secondly how cleavage is modelled.

\subsubsection{Grain Growth}

Researchers have used a range of methods to represent ${ }^{254}$ polycrystalline structures in three dimensions. Voronoi ${ }^{255}$ tessellation has been a common method to model these ${ }^{256}$ crystal structures $[45,46]$. However, the CA uses a differ ${ }^{257}$ ent process to represent the individual crystals. A num- ${ }^{258}$ ber of random nuclei are placed within the CA domain ${ }^{259}$ and the nuclei grow over a number of iterations, to form ${ }^{260}$ a polycrystalline structure. Figure 1 provides a two $\mathrm{di}^{261}$ mensional diagrammatic view of how the grain growth ${ }^{262}$ process takes place.

\begin{tabular}{|c|c|c|c|c|}
\hline & & & & \\
\hline & & $\overline{0}$ & & \\
\hline & 0 & 0 & 0 & \\
\hline & 20 & ) & & 3 \\
\hline & 0 & 0 & & \\
\hline \multicolumn{5}{|c|}{$\begin{array}{l}\text { Grain Nuclei } \\
\text { Initialised }\end{array}$} \\
\hline & & $\overline{4}$ & & \\
\hline & 0 & 4 & 4 & \\
\hline & 0 & 0 & 4 & \\
\hline 5 & & 2 & \begin{tabular}{l|l}
0 & 8 \\
\end{tabular} & \\
\hline & & & & \\
\hline
\end{tabular}

Propagation

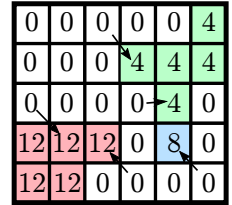

Propagation

\begin{tabular}{|c|c|c|c|c|c|}
\hline 0 & -1 & 4 & 4 & 4 & 4 \\
\hline 0 & 0 & 4 & 4 & 4 & 4 \\
\hline 12 & 12 & 0 & 4 & 4 & 0 \\
\hline 12 & 12 & 12 & 0 & -8 & 8 \\
\hline 12 & 12 & 12 & 12 & 8 & 8 \\
\hline
\end{tabular}

Propagation

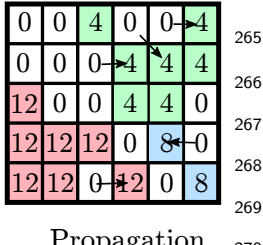

Propagation 270

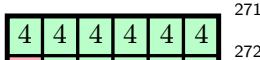
\begin{tabular}{l|l|l|l|l|l|l|l|}
12 & 12 & 12 & 8 & 8 & 8 \\
\hline
\end{tabular} \begin{tabular}{l|l|l|l|l|l|l|}
\hline 12 & 12 & 12 & 12 & 8 & 8 \\
\hline
\end{tabular} Final Grain Structure

Figure 1: Two dimensional example highlighting the process by ${ }^{276}$ which the polycrystalline granular structure is generated within the277 CA grid. The example shows three grain nuclei $(4,8,12)$ and ignores $_{278}$ the grain boundaries.

The entire CA space, or grid is given a value of 0 that ${ }^{280}$ represents a liquid state of the cell. Within the grid $\mathrm{a}_{282}$ random selection of cells are chosen, with each cell repre- ${ }_{283}$ senting a different grain number. At this point each grain ${ }_{284}$ number is also given a randomly chosen orientation. The ${ }_{285}$ grains are subsequently grown from each of the cell nuclei ${ }_{286}^{205}$ over a number of steps. Each step any liquid cell has $\mathrm{a}_{287}$ chance of accepting the cell state of a cell in its neigh- ${ }_{288}$

bourhood. A Moores neighbourhood is used, shown in two dimensions in Figure 2.

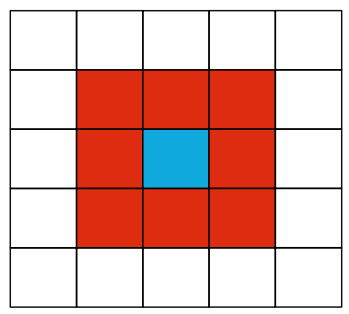

Figure 2: Two dimensional Moores neighbourhood. The state of the central cell shown in blue, is only dependant on its eight surrounding cells shown in red. In three dimensions the Moores neigbourhood is 26 cells large.

The Moores neighbourhood in three dimensions uses the surrounding 26 cells. This process is repeated until the entire CA grid is full and no cells of a liquid state are left. This simple process can lead to a number of differing polycrystalline structures, shown in Section 3. With each of these cells having a different orientation and preferential cleavage plane. Finally the cells that border two or more grains are considered to be grain boundaries, the cell states are altered once the full domain is populated with grains. As part of the grain growth procedure imperfections can be placed with the structure. The simple nature of the method means this can be done by altering the state of specific cells.

\subsubsection{Cleavage}

The cleavage process, based on Gilman [47] suggests that the work required for cleavage is based on the surface fracture energy, $\gamma_{h k l}$. That is, if the normal stress on the crystallographic plane $t_{h k l}$ multiplied by the distance required to break the atomic bonds, is greater than this surface energy, cleavage will occur. The distance to break the atomic bonds is taken as the atom diameter in the cleavage plane, $a_{0}$. The condition for cleavage to occur is therefore,

$$
t_{h k l} \geq \frac{\gamma_{h k l}}{a_{0}},
$$

where $t_{h k l}$, the normal stress on the crystallographic plane is related to the stress tensor $t^{c}$ orientated in the crystals coordinate system through, $t_{h k l}=\boldsymbol{n}_{h k l} \cdot t^{c} \cdot \boldsymbol{n}_{h k l}$. With $\boldsymbol{n}_{h k l}$ being the normal for the hkl plane. Three crystallographic planes are considered, $\{100\},\{110\}$, and $\{111\}$, with cleavage occurring on the weakest plane. The weakest plane is considered to be the plane that maximises the normal stress, $t_{h k l}^{\max }$.

Within this digital framework this cleavage process is completed through a similar step-by-step process to that described in Section 2.2.1, with the fractured cell growing along a preferential cleavage plane for a number of steps. This preferential cleavage plane differs from grain to grain and is dependant on the initial grain orientation assigned 
during the grain growth process. Along with the grain 315 number associated with each cell, they also have a cell ${ }_{316}$ state, that describes the current condition of the cell. A full list of cell states is shown in Table 2. They include a fractured and intact grain boundary, an intact cell within a grain and flank or edge cracks along the hkl planes.

\begin{tabular}{l|c}
\hline \hline Cell State & Value \\
\hline Intact grain boundary & 2 \\
Fractured grain boundary & 1 \\
Intact cell & 0 \\
100 Flank crack & -1 \\
100 Edge crack & -2 \\
110 Flank crack & -3 \\
110 Edge crack & -4 \\
111 Flank crack & -5 \\
111 Edge crack & -6 \\
\hline \hline
\end{tabular}

Table 2: Possible cell states within the digital framework.

At the start of the simulation almost every cell wills18 exist in states 2 and 0 , with some distribution of frac-319 tured cells throughout the grid. For cleavage to occurs20 the cleavage criteria, Equation 7, must first be met. This321 means that the mean stress within the grain must have322 enough energy to overcome one of the three planes sur-323 face energies. If this criteria is met cleavage occurs on 324 the weakest of the three planes. The process to find the325 weakest plane is shown in Algorithm 1:

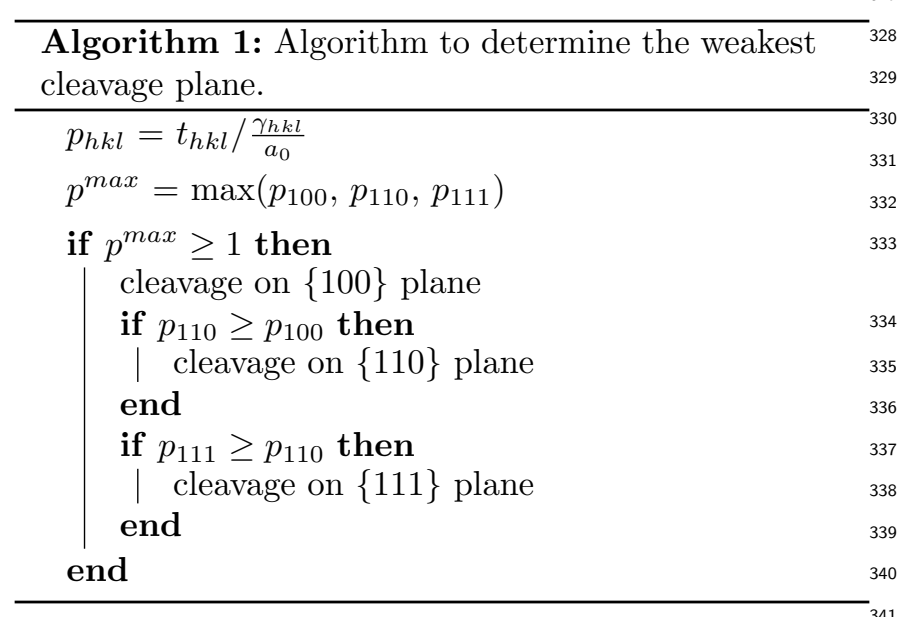

Cleavage occurs through a stepping process, similar to ${ }^{342}$ that used to generate the granular structure. An intact ${ }_{344}^{343}$ cell can change state and become a fractured cell if (i) it ${ }^{344}$ is adjacent to a fractured cell, (ii) it lies on the cleavage ${ }_{346}$ plane, and (iii) the cleavage criteria is met.

The total number of cleavage steps per time step is dependant on a crack propagation speed, the time step $_{348}$ and the cells per unit length. If a material starts with ${ }_{349}$ a number of fractured cells, over a number of time steps ${ }_{350}$ where the cleavage stress tensor is large enough, cleavage occurs and a number of cleavage planes are generated. ${ }_{352}$
A two dimensional example of the process is shown in Figure 3.

\begin{tabular}{|c|c|c|c|c|c|c|c|c|c|c|c|c|c|c|c|c|c|c|c|c|c|c|c|}
\hline \multicolumn{12}{|c|}{ Initial } & \multicolumn{12}{|c|}{ After a number of steps } \\
\hline 1 & 1 & 1 & 1 & 1 & 2 & 1 & 1 & 1 & 1 & 1 & 1 & & \begin{tabular}{|l|l|}
1 & 1 \\
\end{tabular} & 1 & 1 & 1 & 2 & 1 & 1 & 1 & 1 & & 1 \\
\hline-1 & 1 & 1 & 1 & 2 & 2 & 1 & 1 & 1 & 1 & 1 & 1 & & $-1-1$ & -1 & -1 & 2 & 2 & -3 & 1 & 1 & 1 & 1 & 1 \\
\hline 1 & 1 & 1 & 1 & 2 & 1 & 1 & 1 & 1 & 1 & 1 & 1 & & \begin{tabular}{l|l|}
1 & 1 \\
\end{tabular} & 1 & 1 & 2 & 1 & 1 & 3 & 1 & 1 & 1 & 1 \\
\hline 1 & 1 & 1 & 1 & 2 & 1 & 1 & 1 & 1 & 1 & 1 & 1 & & \begin{tabular}{l|l}
1 & 1 \\
\end{tabular} & \begin{tabular}{|l|}
1 \\
\end{tabular} & 1 & 2 & 1 & 1 & 1 & 33 & 1 & 1 & 1 \\
\hline 1 & 1 & 1 & 1 & 2 & 1 & 1 & 1 & 1 & 2 & 2 & 2 & 1 & $\begin{array}{lll}1 & 1 \\
\end{array}$ & 1 & 1 & 2 & 1 & 1 & 1 & 11 & 2 & 2 & 2 \\
\hline 2 & 2 & 2 & 2 & 2 & 1 & 1 & 2 & 2 & 2 & 1 & 1 & 2 & \begin{tabular}{l|l}
2 & 2 \\
\end{tabular} & 2 & 2 & 2 & 1 & 1 & 2 & 2 & 2 & 1 & 1 \\
\hline 1 & 1 & 1 & 1 & 2 & 2 & 2 & 1 & 1 & 1 & 1 & 1 & 1 & \begin{tabular}{|l|l|}
1 & 1 \\
\end{tabular} & 1 & -1 & 2 & 2 & 2 & 1 & 1 & -3 & 1 & 1 \\
\hline 1 & 1 & 1 & 1 & 1 & 1 & 2 & 1 & 1 & 1 & 1 & 1 & 1 & \begin{tabular}{l|l}
1 & 1 \\
\end{tabular} & -1 & 1 & 1 & 1 & 2 & 1 & 1 & \begin{tabular}{|l|l|} 
\\
\end{tabular} & 1 & 1 \\
\hline 1 & 1 & 1 & 1 & 1 & 1 & 2 & \begin{tabular}{|l|}
2 \\
\end{tabular} & 1 & -3 & 1 & 1 & 1 & $\begin{array}{ll}1 & -1 \\
\end{array}$ & 1 & 1 & \begin{tabular}{|l|}
1 \\
\end{tabular} & 1 & 2 & 2 & 1 & -3 & 1 & 1 \\
\hline-1 & 1 & 1 & 1 & 1 & 1 & 1 & 2 & 1 & 1 & 1 & 1 & & $11^{\prime}$ & 1 & 1 & 1 & 1 & 1 & 2 & 1 & 1 & 11 & 1 \\
\hline
\end{tabular}

Figure 3: Two dimensional example highlighting the cleavage process. A number of fractured cells exist within the model which propagate along the preferential cleavage planes within the grains(marked with a dashed line). If a crack crosses a grain boundary it will continue to cleave however it will propagate along the new preferred cleavage plane.

It must be noted that this method of modelling cleavage has its simplifying assumptions, the mesoscale nature of the problem means that it exists somewhere in-between molecular dynamics and continuum scale methods. The method has yet to include micro-plasticity that is observed at the crack fronts, during brittle fracture and so critical stresses of the material itself do not correspond exactly to those seen by Gilman [47]. A calibration of the critical stress parameters seen in real materials and those in the applications is required.

This subsection has provided a description of how polycrystalline materials are generated using the $\mathrm{CA}$ and how the brittle fracture of these materials takes place. The following sections will provide a summary of the parallel implementation of the CA, before describing the coupled nature of the two methods, CA and FEM for the solution of fracture using a multiscale strategy.

\subsubsection{Parallel Implementation}

The CA is implemented using coarray Fortran (CAF), that has been part of the Fortran standard since 2008. CAF uses the single program multiple data model, which at runtime creates a series of images that execute the same code. CAF enables remote read and write access from each image, with the execution segments acting on an asynchronous basis. Global barriers exist, named image control statements that are utilised to ensure correct data management and avoid race errors. Using this methodology CASUP has run problems with around 80 billion cells on around 32,000 cores. Detailed description of the implementation of the Coarrays in CASUP can be found in $[24,34]$.

\subsection{Multiscale Modelling : CAFE}

The methodologies used to model the macroscale and mesoscale have been described. This section subsequently provides a summary of how the scales and packages interact and the key transfer of data between the two. As 
with a number of multiscale modelling approaches [48] localisation and homogenisation techniques are used to couple the two scales, and will be described in more detail. An implementation of the CAFE model is provided in Figure 4, with a description of it below.

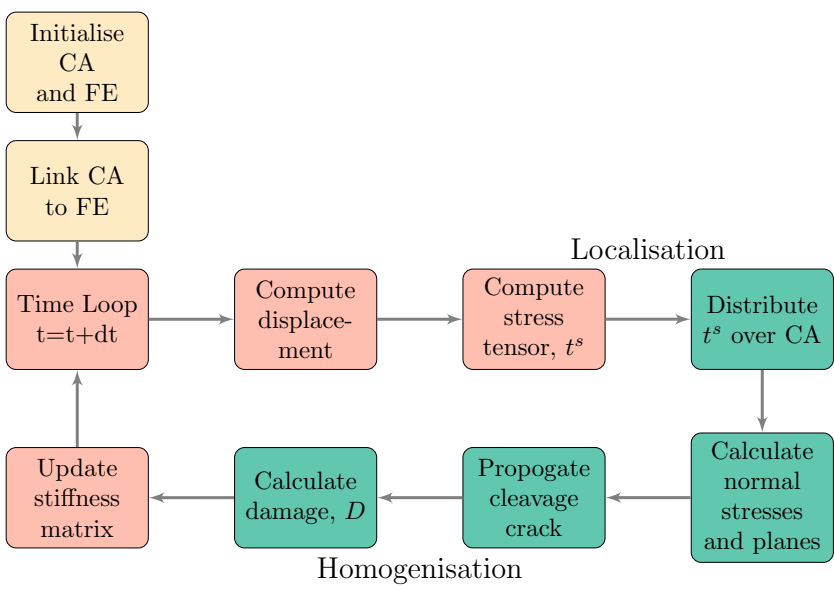

Figure 4: CAFE implementation highlighting the key steps, with processes run by both applications in yellow (light), those run by ParaFEM in red (darker) and CASUP in green (darkest). The steps over which the localisation and homogenisation occur are labelled.

The initialisation phase that takes place, sets up the problem. A file based I/O is used to read in the mesh information for ParaFEM, this includes three files, a definitions file containing numerical parameters and material properties, a file containing the element steering arrays and a file containing the nodal coordinates. The master processor reads the file and distributes the steering array and coordinates among the MPI processes. The CA requires only a number of values to define its space and composition. The coordinates of its origin and a vector with the length of each orthogonal side defines the physical dimensions of the grid. The CA grid must overlap the FE if only partly as only CA that are with FE's are378 involved in the calculation of fracture. The dimensions 379 of the cellular automata and the size of the coarray that 380 computationally represents the CA, is further defined by ${ }^{381}$ the average grain size and the mean number of cells per382 grain. At this phase within the program the grain growth ${ }^{383}$ process, described in Section 2.2.1, also takes place, gen-384 erating the polycrystalline structure within the CA. $\quad 385$

The linking between the two applications takes place 386 by coupling clusters of $\mathrm{CA}$ that exist within a $\mathrm{FE}$ ele-38т ment region to the $\mathrm{FE}$ element itself. As is common with 388 multiscale methods the element and cell dimensions areзsя of drastically different sizes and so a large number of CA390 can exist within each FE. Figure 5 provides a two di-391 mensional example of the clustering and linking within392 the application. The linking is a quick effective method. ${ }^{393}$ A characteristic length is defined by the user, Lc that394 defines the radius of a sphere whose origin is at the el-395 ements centre. Any automata, no matter the cell state,396 that exists within the elements characteristic length is 397

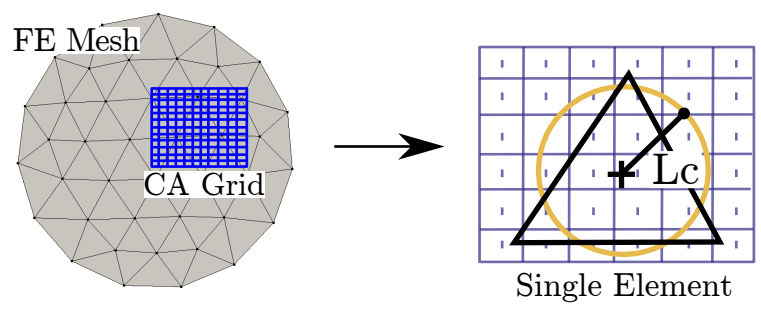

Figure 5: An example of the linking process in two dimensions for an embedded CA grid in FE mesh. A automata is associated with an element if it is within its characteristic length, Lc, highlighted in the figure.

assigned to the element. Subsequently, each element contains a collection of automata whose states impact the elements macro properties. After the initialisation and linking phases, the time loop can commence. The solution to the FE problem described in Section 2.1.1 is used to generate the stress tensor for each element. At this point the stress tensor of each element is distributed across the cluster of CA cells that they are associated with. The mean stress of the $\mathrm{FE}$ and the $\mathrm{CA}$ is equal, however the stress is distributed such that higher stress values lie at crack fronts. Localisation techniques such as these are common amongst multiscale approaches. CASUP then uses the CA stress values to determine whether cleavage will occur, see Section 2.2.2. A homogenisation step takes place to update the macro properties of the material with the effects calculated at the mesoscale. A damage variable is calculated that is a ratio of the number of fractured cells to the total number of cells within the $\mathrm{CA}$ cluster or $\mathrm{FE}$.

$$
\text { Damage }=\frac{\text { fractured cells }}{\text { total cells }}
$$

This damage scalar, is used to update the element stiffness. Conceptually as the damage increases the load bearing capacity of the element falls. With the updated Young's Modulus of the element, the stiffness matrix is recalculated. This loop continues until the material fails or the user defined time limit has been reached. Extreme events such as cracks perpendicular to the loading force that result in the element's stiffness falling to zero are not captured by such a model. However, these cases are rare, the heterogeneous grains that have random orientations mean it is unlikely a group of adjoining grains, that impact a finite element's stiffness, have cleavage planes all running in the same direction. The comparison of the simulation with experimental results can be used to calibrate the model, but as a problem tends towards the extreme events, such as that described, the model results will drift away from the experimental values, as is typical with a number of numerical methods.

The application described comes as part of ParaFEM that is available at the git repository under a BSD licence. 
The mini-app is labelled xx20 within the "src/program-431 s/dev directory.

\section{Results}

The framework is demonstrated through the use of $\mathrm{a}^{436}$ simple test problem that considers a cantilever beam un-437 dergoing forced vibration that ultimately leads to brittle ${ }^{438}$ fracture within the material. The test case aims to highlight the capabilities of the CAFE model with the added ${ }^{439}$ capacity to model transient problems and further high-440 light the key parameters to consider when continuing to ${ }^{441}$ develop and use the application.

\subsection{Problem Statement}

In this problem the brittle fracture of a polycrystalline $e^{45}$ material undergoing forced vibration is analysed. Firstly ${ }^{446}$ a range of loads are applied to identify loading regions ${ }^{447}$ over which fracture will occur. After selecting one of ${ }^{448}$ these loading factors an in depth analysis provides details ${ }^{449}$ of the effect at the mesoscale and macroscale. Figure $6^{450}$ provides a schematic of the problem test case.


Figure 6: Computational domain of the problem, the boundary conditions are shown on the right without the FE mesh. The CA region is highlighted within the $\mathrm{FE}$ domain with a snapshot of a grain structure on the right.

\subsubsection{Modelling at the macroscale}

The elastic beam has dimensions of $0.01 \mathrm{~m}, 0.05 \mathrm{~m}$ and $0.01 \mathrm{~m}$ in the $\mathrm{x}, \mathrm{y}$ and $\mathrm{z}$ directions respectively. The CA zone is embedded within the elastic beam at the mid point in the $\mathrm{Y}$ direction. The FE continuum has a Young's modulus of $200 \mathrm{GPa}$, a density of $7874 \mathrm{~kg} / \mathrm{m}^{3}$ and a poisson's ratio of 0.3 , which corresponds to the material properties of iron. The beam is fixed at its base and forced with a constant load at its tip. Quadratic 20 noded elements are used throughout the series of tests, using 5000 elements for the initial loading sweeps and approximately ${ }^{461}$ 78,000 for the analysis of the fracture case.

The choice of length scale and time scale, for multi- ${ }^{463}$ scale methods, can be challenging, and highly problem ${ }^{464}$ dependent [49]. The time scales for cleavage are in the orders of $10^{-6} s$ to $10^{-3} s$ respectively. In comparison engineering time scales can range anywhere from $10^{-1} s$ to $10^{4} s$, when using continuum methods. Within this case a time step of $0.0001 s$ is used, which means a possible fractured cell will cleave the entire $\mathrm{CA}$ zone within one time step. Within this test case the brittle fracture step is only completed once at $0.002 s$.

\subsubsection{Modelling at the mesoscale}

The CA zone is a cube with sides of length $0.01 \mathrm{~m}$. The CA zone is populated with approximately $100 \times 10^{6}$ cells and 1000 grains, which corresponds to $1 \times 10^{5}$ cells per grain, which has been found by Shterenlikht and Margetts [24] to provide grid independent results for a quasi-static analysis. The mean grain diameter is $0.001 \mathrm{~m}$ which is a factor of two larger than the average grain size for iron. In this mechanistic methodology the crack propagation speed is set at $1000 \mathrm{~m} / \mathrm{s}$. The critical stresses are from Gilman [47], set at 1.05, 1.25 and $4.9 \mathrm{GPa}$ for the $\{100\}$, $\{110\}$ and $\{111\}$ cleavage planes respectively.

\subsection{Initial sweep}

The initial loading sweeps, involved ramping up the load to determine the range of load values at which fracture will occur. Figure 7 presents the displacement of the cantilever tip against time. It highlights when cracks are initialised at $0.0025 \mathrm{~s}$ and how differing loads and crack formations, result in different macroscopic profiles of displacement. Figure 8 shows the fracture volume as a percentage of the total number of cells, with the loads corresponding with those in Figure 7.

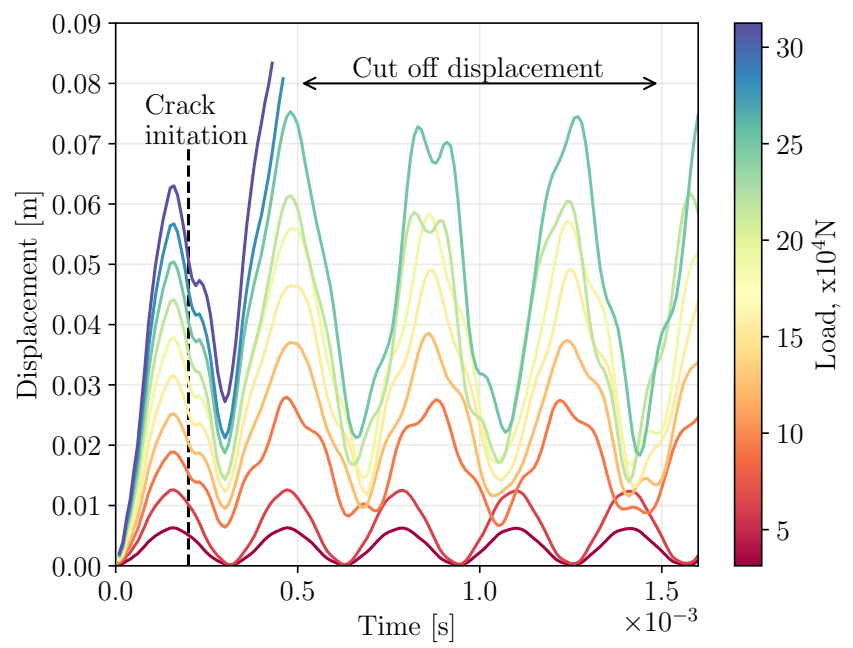

Figure 7: Displacement time plots for a range of loads. The cut off displacement is shown at $0.08 \mathrm{~m}$ and the crack initiation at $0.0025 \mathrm{~s}$.

The figures highlight that for brittle fracture to occur at the specified time step the load must be approximately $93.8 k N$ or above. The displacement time plot for the values below this follow a perfectly elastic response with 


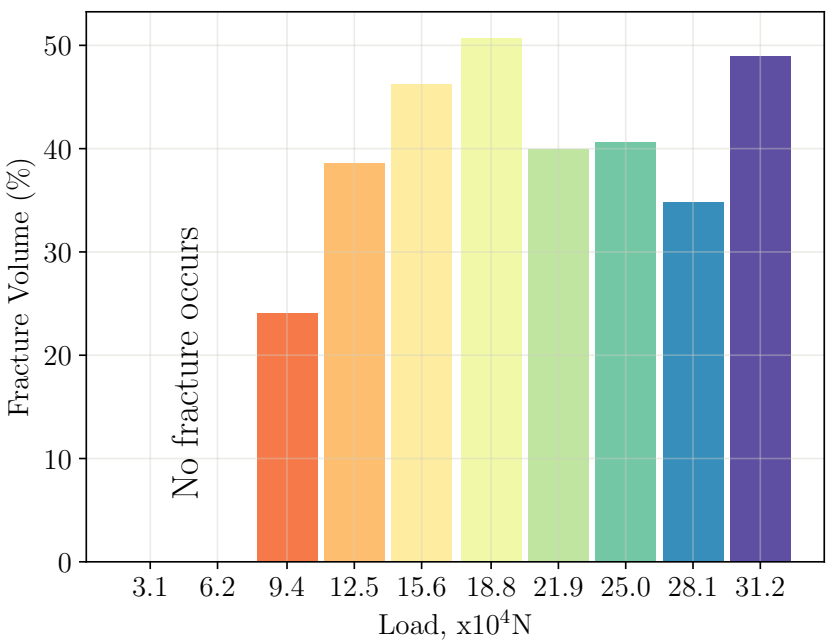

Figure 8: The percentage fracture volume for each of the load cases.

no brittle fracture taking place. As the load increases from $93.8 \mathrm{kN}$ the percentage of fracture volume increases, however the difference in fracture volume after $125 k N$ is suggested to be due to variation in the grain structure for each of the simulations run. The following section will provide further information into the mini-app and the details of how different scales react.

\subsection{Analysis and Discussion}

Considering the load, $94 k N$, the finite element mesh was refined from 5000, 20 noded, hexahedral, quadratic elements to approximately 78,000 . This larger test case was run a number of times, with each run on 120 cores. The statistical behaviour of brittle fracture and material properties can be integrated within the miniapp. The polycrystalline structure of the material is randomly generated, and so running the same application on the same machine can result in a range of grain orientations, sizes and shapes. Figure 9 presents the polycrystalline structure formed using the application for a number of the test cases run.

It is clear from Figure 9 that there is an inherent varia-502 tion within the generation of the polycrystalline structure ${ }_{503}$ and hence the cleavage and formation of fracture planes 504 within the structure. Although this variation exists, the ${ }_{505}$ localisation techniques tend to smear this variation out 506 on the continuum scale such that the deformation and ${ }_{507}$ stress tensors at this engineering scale are similar for all ${ }_{508}$ the runs. This variation leads to differing micro crack $_{509}$ formations and ultimately differing macro cracks within $n_{510}$ the structure. The test case was run ten times and Fig- ${ }_{511}$ ure 10 shows the micro crack formulations for six of these ${ }_{512}$ runs.

It can be seen that the formation of the micro cracks ${ }_{514}$ differs from case to case, depending on the initial poly-515 crystalline grain structure and orientation. The colors of $f_{516}$ the micro cracks represent the plane in which cleavage ${ }_{517}$

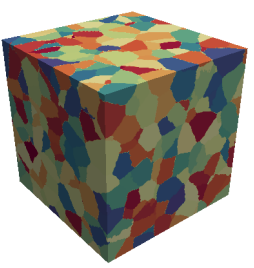

(a)

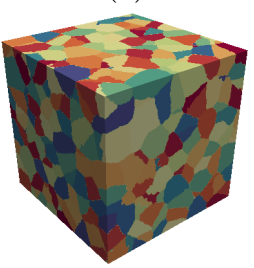

(d)

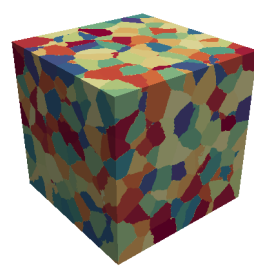

(b)

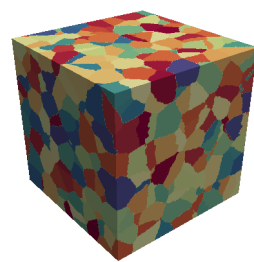

(e)

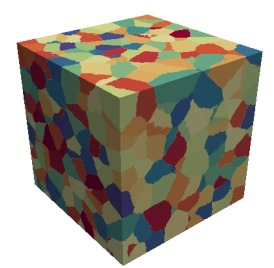

(c)

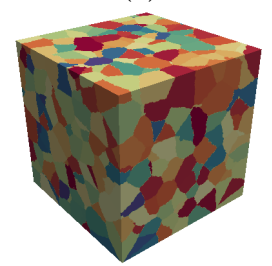

(f)
Figure 9: Grain distribution from six simulations running on the same machine and the same number of cores, each colour represents a different grain with 1000 grains.

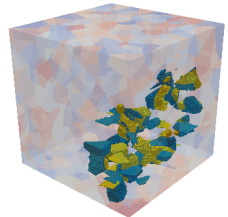

(a)

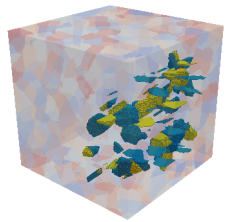

(d)

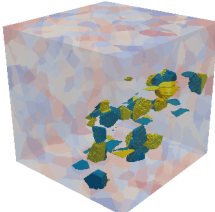

(b)

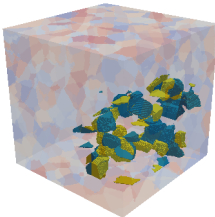

(e)

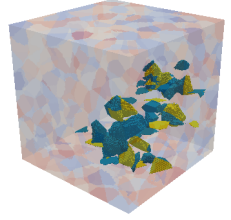

(c)

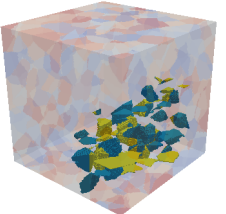

(f)
Figure 10: Figures showing the variation in micro crack formulation for the same job. The green and yellow cracks show cleavage in the $\{100\}$ and $\{110\}$ planes respectively.

has occurred. The green represents cleavage in the $\{100\}$ plane and the yellow in the $\{110\}$. There is no cleavage on the $\{111\}$ plane due to the significantly larger cleavage stress required.

Figure 11 highlights how the combination of micro cracks leads to the formulation of a macro crack.

A red band has been drawn on the figure to highlight the orientation and region in which the microcracks have merged to generate a macro crack. The variation in both the effect at the mesoscale and macroscale are shown in Figures 12 and 13 that again show the percentage of fracture volume and the tip displacement respectively.

Figure 12 shows that the percentage fracture volume can range by around $15 \%$ from a maximum of $37 \%$ in analysis (g) to a minimum of $22 \%$ in analysis (d). Comparing this to the micro cracks formed in Figure 10 there is a noticeable difference in density of fracture in 

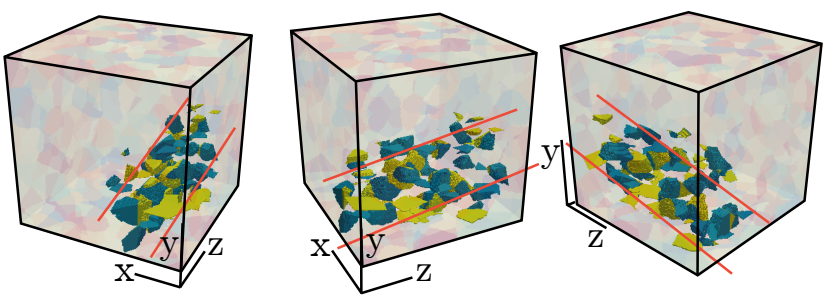

1

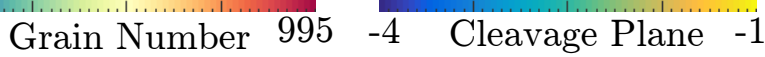

Figure 11: Three views rotated, to show the three dimensional micro cracks generated, the green and yellow represent the the 100 and 110 cleavage planes respectively. A red band has been drawn to highlight the formation of a macro crack.

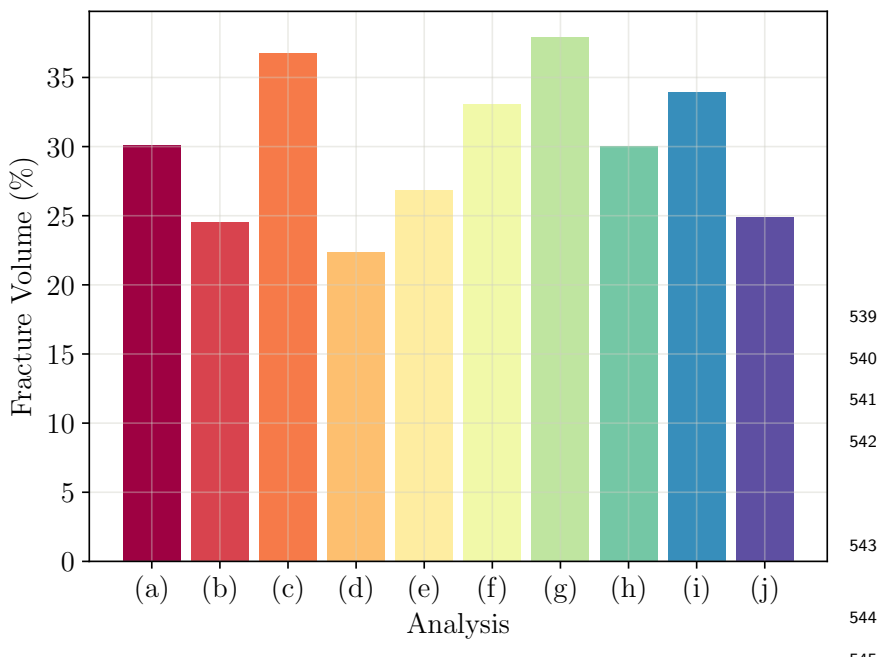

Figure 12: The fracture volume percentage for the 10 analyses run.546 The colors associated with the fracture volume are consistent with h $_{547}$ Figure 13.

analysis (d) and (g). From Figure 13, it appears that ${ }^{549}$ the level of fracture correlates with the peak deflection, ${ }^{550}$ as at the second peak after fracture analysis $(\mathrm{g})$ has $\mathrm{a}^{551}$ larger peak deflection value than analysis (d). The overall ${ }^{52}$ impact of the mesoscale model at the continuum scale ${ }^{53}$ appears in the form of differing tip displacement profiles ${ }^{54}$ and peak deflections.

After considering the mesoscale and macroscale an ex-550 ample of the full work flow is highlighted in Figure 14.557 Figure (a) shows the displacement magnitude in meters 558 with the impact the mesoscale model has had. It can be ${ }^{559}$ seen that the damage caused by the fracture has caused ${ }^{560}$ a softening of the material and a loss of load bearing ca-561 pacity. Figure (b) shows where the fracture sits within ${ }^{562}$ the finite element structure. Figure (c) shows the con-563 tinuum that has been warped by a scale factor of 1 . A A54 $^{56}$ clear macrocrack can be seen where the mesoscale model ${ }^{565}$ exists.

The fracture at the mesoscale has caused a significant ${ }^{566}$ drop in the load bearing capabilities of the material and567 hence a region where the finite elements are deforming568

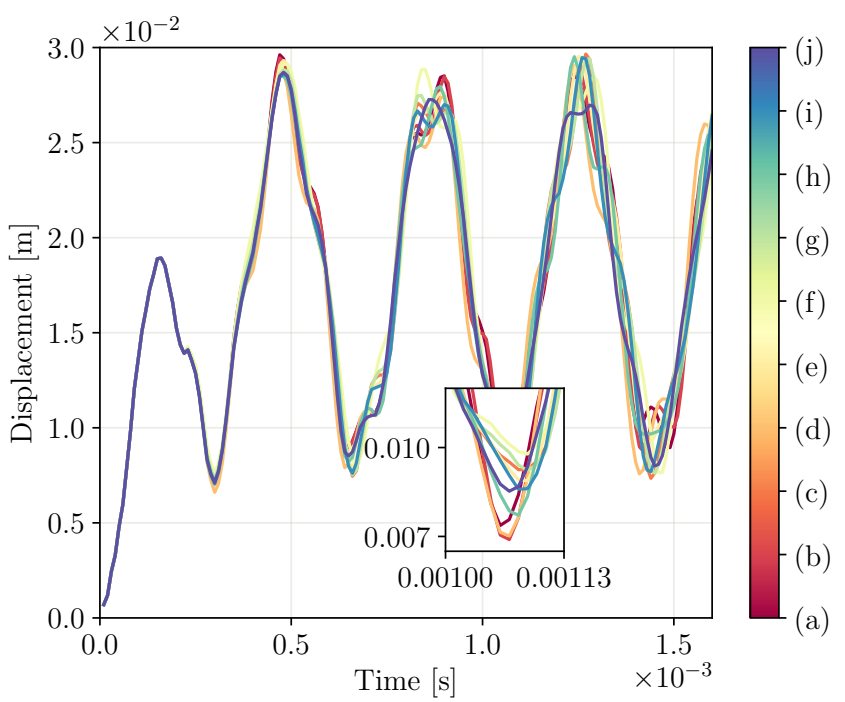

Figure 13: The displacement at the tip of the beam for the 10 jobs run. The inset figure highlights the differences in peak deflection for each of the jobs.

significantly. This section has provided an example test case exploring some of the parameters associated with the miniapp, and how it can be used to explore the grain orientation design space.

\section{Parallel Performance}

Multiscale modelling approaches are well documented in the literature, however they often fail to acknowledge the computational expense. This section highlights the scalability of this application on high performance computing systems.

\subsection{Computational Expense}

The application was compiled on ARCHER [50], the UK's national computing service, which is a Cray XC30 machine. The nodes are made up of two 12 core E5-2697 Ivy Bridge processors with a clock speed of $2.7 \mathrm{GHz}$. The compute nodes are linked using the Cray Aries interconnect, with each compute node having 2 non-uniform access (NUMA) regions with 32 GB of memory on each processor. Both packages were complied using version 8.5.8 of the Cray compiling environment. Profiling information was collected for the simulations using the tracing tool, CrayPAT.

The test case for both the strong and weak scaling is based on that shown in Section 3. Three finite element meshes and three CA grids were generated for the initial analysis and the strong scaling studies. Full details of the FE meshes and CA grids can be found in Table 3 .

\subsubsection{Performance Analysis}

The initial performance analysis considers the breakdown of execution time into the four major components. 


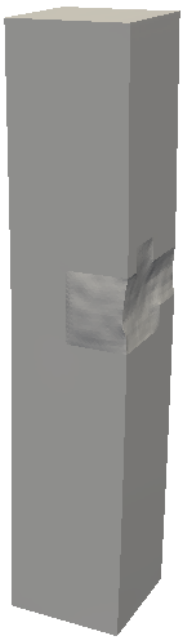

(a)

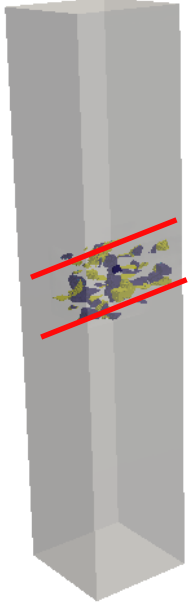

(b)

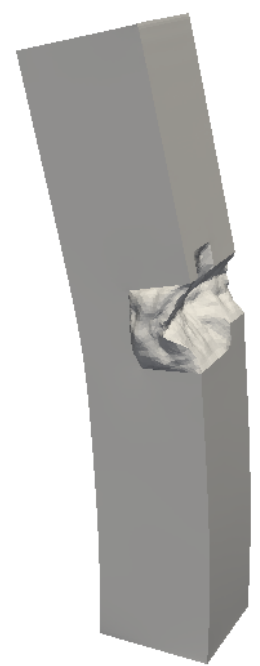

(c)

Figure 14: From left to right the figures show, (a) the displacement at the macroscale, (b) the cleavage planes inset within the FE model and (c) the FE model, warped by its displacement vector.

\begin{tabular}{c|ccc}
\hline \hline Mesh & $\begin{array}{c}\text { \# Nodes } \\
\left(\mathrm{x} 10^{6}\right)\end{array}$ & $\begin{array}{c}\text { \# Equations } \\
\left(\mathrm{x} 10^{6}\right)\end{array}$ & $\begin{array}{c}\text { \# of CA } \\
\text { Cells }\left(\mathrm{x} 10^{6}\right)\end{array}$ \\
\hline $\mathrm{A}$ & 0.3 & 1 & 50 \\
$\mathrm{~B}$ & 2.5 & 7.7 & 500 \\
$\mathrm{C}$ & 20 & 60 & 5000 \\
\hline \hline
\end{tabular}

Table 3: Summary of the three FEM meshes and CA grids used for the initial breakdown study and analysing the strong scalability of the application.

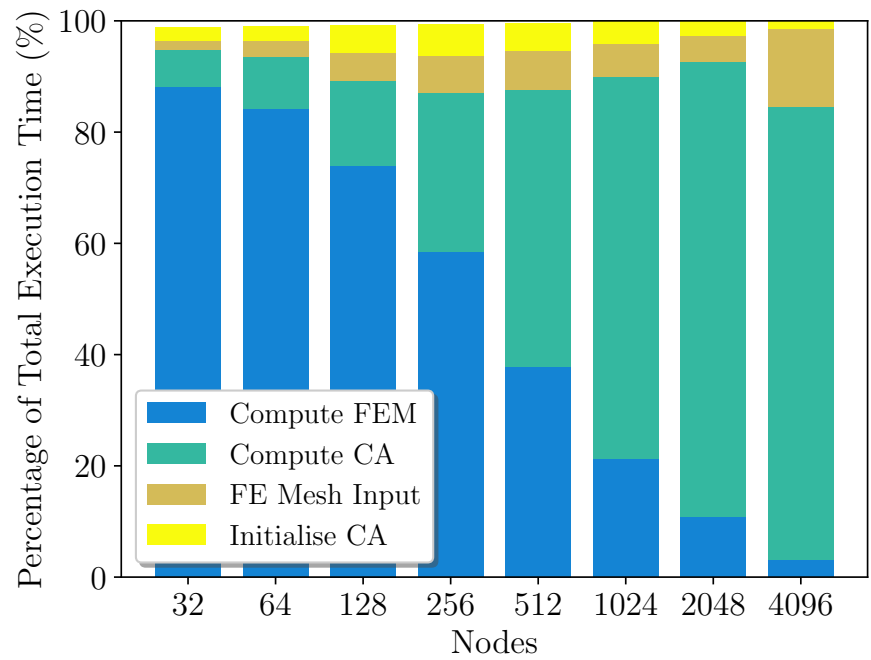

(a) The percentage of total execution time spent in the major components of the application.

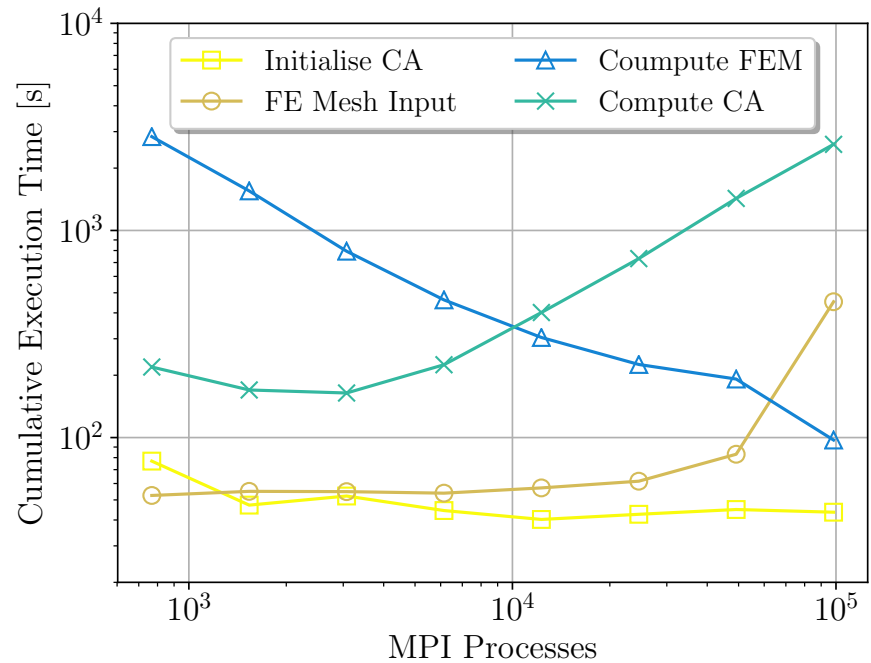

(b) The execution time for each major component in the application.

Figure 15: The initial cost estimation of the application using Mesh C from Table 3.

From Figure 15a, it can be seen that the initialisation time for ParaFEM is constant up to approximately 25,000 cores before increasing to a significant proportion of the problem execution time, representing around $14 \%$ of the solution time on approximately 100,000 cores. For this problem the time spent solving each scale is initially distributed heavily towards the FE before inverting, with the CA taking up approximately $81 \%$ on 100,000 cores, whilst the FE represents approximately $3 \%$.

When two packages are coupled sequentially, each existing on different processor, the maximum computational efficiency that can be achieved is 0.5. However, in this case both packages share the same computational resources. The data arrays for each domain are distributed to the available processors. Therefore, each processor contains decomposed data from both domains with mappings link- 




(a) Speedup of the application time excluding the initialisation time for CASUP and the read in time for ParaFEM.

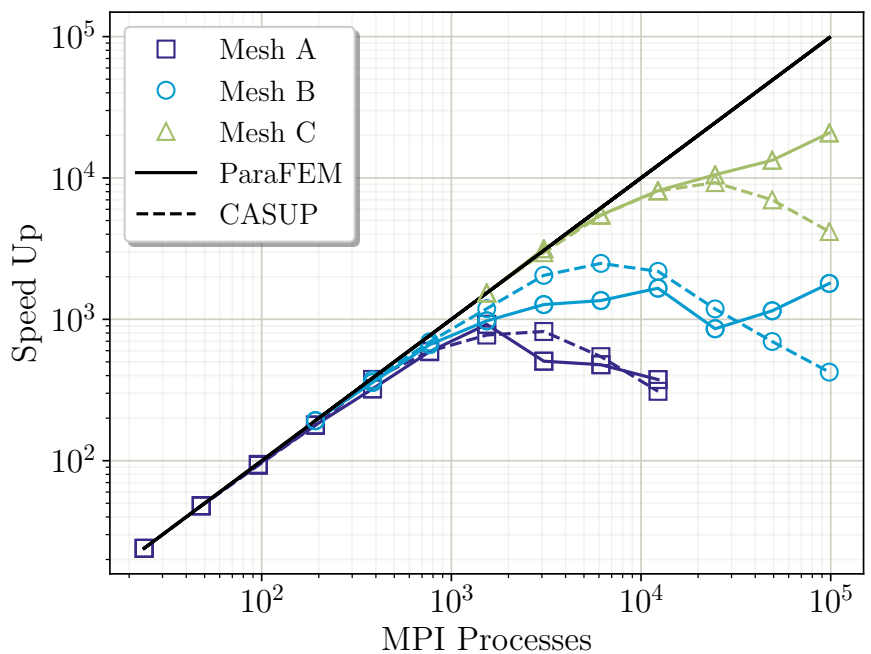

(b) Speedup of ParaFEM and CASUP for each of the three meshes used.

Figure 16: Strong scaling results of the application.

ing information that needs to be coupled. The approach 623 means that although the ratio of execution time may vary624 (rastically between the two packages, the computational $6_{22}$ is high, with the processors rarely being idle.620 The following subsections will provide further detail on $_{627}$ the strong and weak scaling of the mini-app. tion, excluding setup time, whilst Figure 16a presents the ${ }^{63}$ speedup of CASUP and ParaFEM independently.

\section{.}

.

\section{.}

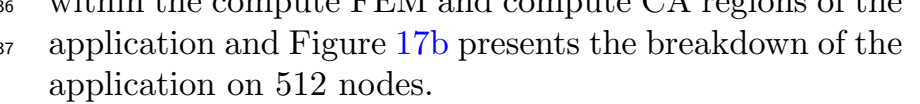
application on 512 nodes.



(a) Percentage breakdown of the computational time, ignoring the initialisation and read in time.



\author{
FEM-IO read \\ FEM-math ops. \\ All other \\ $\square$ FEM-gather \\ FEM-scatter \\ CA-memory barrier \\ CA cleavage \\ FEM to CA linking
}

(b) Average percentage of time spent within significant functions for the application run on 512 nodes.

Figure 17: Weak scaling results of the application.

The time step for the weak scaling is constant, so the physical time remains the same for each simulation. Subsequently, it can be seen that the percentage of CPU time spent in each of the regions is consistent from 24 cores to 12288 cores. The size of the problem reaches a maximum at 512 nodes with approximately 50 million nodes in the 


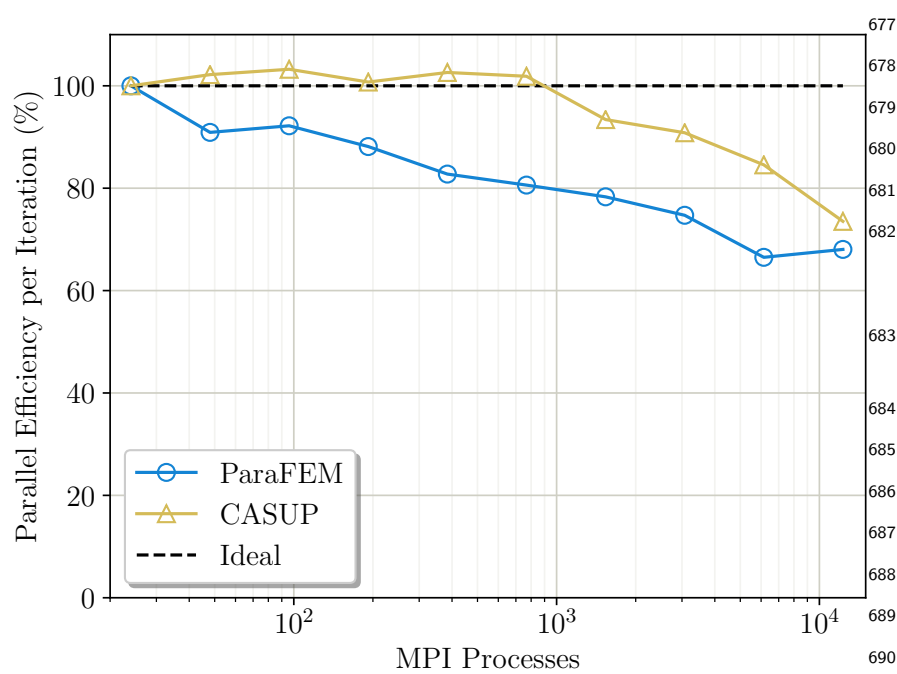

(a) Parallel efficiency of ParaFEM and CASUP, per total num- ${ }^{691}$ ber of PCG iterations and steps respectively.

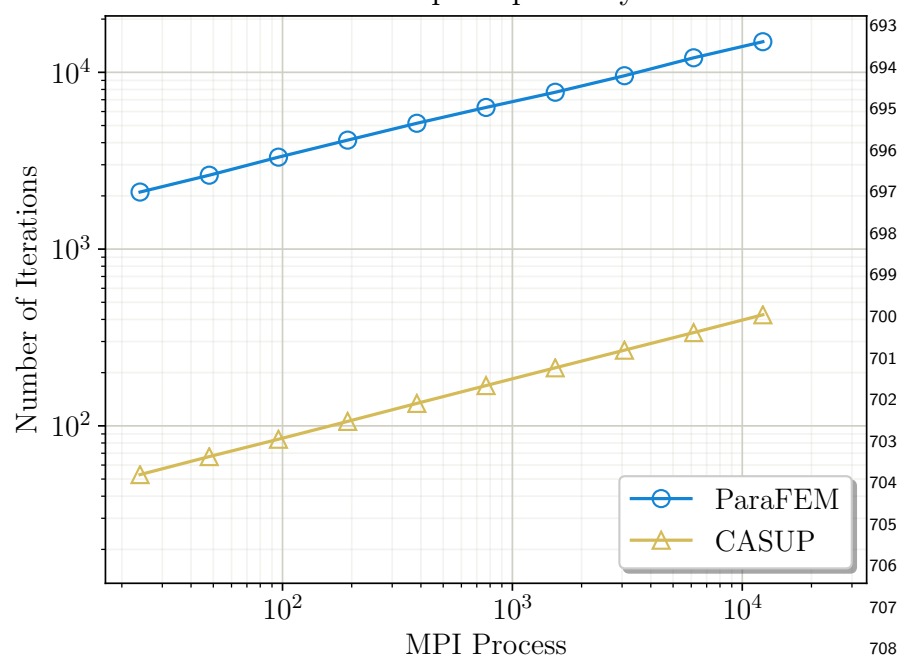

(b) Increase in the number of PCG iterations and steps as the ${ }^{709}$ problem size grows.

Figure 18: Weak scaling results of the application.

FE domain and 5 billion cells in the CA domain. The 6 reak down of the application shows that for this test664 IO read and FEM to CA linking). The solution to the $\mathrm{FE}_{667}$ problem is broken down into time spent in mathematical 668 operations, FEM-math ops. (18.7\%) and time spent in669 related subroutines, FEM-gather and FEM-scatter670

Figures $18 \mathrm{a}$ and $18 \mathrm{~b}$ present the parallel efficiency of 672 each of the computational stages, solving the FEM prob-673 lem and iterating the $\mathrm{CA}$, and the increase in the number 674 of PCG iterations and steps required as the problem size675 grows.

From Figure 18a it can be seen that CASUP achieves an ideal parallel efficiency to around 1000 cores, and ${ }^{712}$ still remains above $75 \%$ on approximately 12,000 cores. $^{713}$ ParaFEM in comparison, has a more gradual decline in ${ }^{714}$

\section{7} 679 .

\section{, s. \\ 5. Conclusion}

This paper has presented, for the first time, a multiscale modelling framework for dynamic problems (cyclic loading and impact) where the macroscale model of the engineering component accumulates damage computed by the mesoscale simulation of real physical mechanisms that lead to fracture. A mini-app has been written to demonstrate the use of the software for a simple example problem. The problem involves the fracture of an iron bar undergoing forced vibration. The bar includes a region of cellular automata comprising a randomised configuration of grains at the mesoscale. The results show a good qualitative representation of Mode I fracture in a polycrystalline material. Further calibration of the parameters used to model cleavage is required to provide more exact results. The CAFE mini-app scaled well to approximately 24,000 cores.

The computational model presented in this paper sits at levels 2-3 in NASA's technology readiness scale. The primary goal has been to investigate whether such a simulation strategy could work for the modelling of dynamically loaded materials undergoing fracture and failure in a multiscale framework. The calibration and validation of the physical model is still required, however, it is envisaged that this simulation strategy could be used in the future to design and test new materials; leading to improved performance of components and devices subject to extreme operating conditions.

\section{Acknowledgements}

The authors acknowledge the support of EPSRC and General Electric through grant EP/M507969/1, along with an EPSRC grant EP/N026136/1 and its associated Archer 
Project e515, entitled "GEMS : Geometric Mechanics of 784 Solids".

\section{References}

[1] D. S. Dugdale, Yielding of Steel Sheets Containing Slits, Jour- ${ }_{790}^{789}$ nal of the Mechanics and Physics of Solids 8 (2) (1960) $100{ }^{-}{ }_{791}$ 104. doi:10.1016/0022-5096(60)90013-2.

[2] G. Barenblatt, The Mathematical Theory of Equilibrium ${ }_{793}^{792}$ Cracks in Brittle Fracture, in: Advances in Applied Me- ${ }_{794}$ chanics, Vol. 7, Elsevier, 1962, pp. 55-129. doi:10.1016/ ${ }_{795}$ S0065-2156(08)70121-2.

[3] A. Turon, C. Dávila, P. Camanho, J. Costa, An Engineer- ${ }_{797}^{796}$ ing Solution for Mesh Size Effects in the Simulation of Delamination using Cohesive Zone Models, Engineering Frac- ${ }_{799}$ ture Mechanics 74 (10) (2007) 1665-1682. doi:10.1016/J. ${ }_{800}$ ENGFRACMECH . 2006.08.025.

[4] S. A. Ponnusami, S. Turteltaub, S. van der Zwaag, Cohesive- ${ }^{801}$ Zone Modelling of Crack Nucleation and Propagation in in $_{803}$ Particulate Composites, Engineering Fracture Mechanics $149_{804}$ (2015) 170-190. doi:10.1016/J.ENGFRACMECH . 2015.09.050. 805

[5] I. Simonovski, L. Cizelj, Cohesive Zone Modeling of Inter- ${ }_{806}$ granular Cracking in Polycrystalline Aggregates, Nuclear En- ${ }_{807}$ gineering and Design 283 (2015) 139-147. doi:10.1016/j.808 nucengdes.2014.09.041.

[6] A. Seagraves, R. Radovitzky, Dynamic Failure of Materials ${ }_{810}^{809}$ and Structures, in: Advances in Cohesive Zone Modeling of Dynamic Fracture, Springer, 2009.

[7] R. Radovitzky, A. Seagraves, M. Tupek, L. Noels, A Scal- ${ }_{813}^{812}$ able 3D Fracture and Fragmentation Algorithm Based on $\mathrm{a}_{814}$ Hybrid, Discontinuous Galerkin, Cohesive Element Method, ${ }_{815}$ Computer Methods in Applied Mechanics and Engineering ${ }_{816}$ 200 (1-4) (2011) 326-344. doi:10.1016/J. CMA. 2010.08.014. 817

[8] N. Moës, J. Dolbow, T. Belytschko, A Finite Element Method ${ }_{818}$ for Crack Growth Without Remeshing, International Journal ${ }_{819}$ for Numerical Methods in Engineering 46 (1) (1999) 131150. doi:10.1002/(SICI) 1097-0207(19990910) 46:1<131: $:_{821}$ AID-NME726>3.0.CD;2-J.

[9] J. Oliver, A. Huespe, P. Sánchez, A Comparative Study on ${ }^{822}$ Finite Elements for Capturing Strong Discontinuities: E-FEM $_{824}$ Vs X-FEM, Computer Methods in Applied Mechanics and En- ${ }_{825}$ gineering 195 (37-40) (2006) 4732-4752. doi:10.1016/J. CMA. ${ }_{826}$ 2005.09.020.

[10] R. J. Nuismer, An Energy Release Rate Criterion for $\operatorname{Mixed}_{828}^{827}$ Mode Fracture, International Journal of Fracture 11 (2) (1975) ${ }_{829}$ 245-250. doi:10.1007/BF00038891.

[11] G. Vigueras, F. Sket, C. Samaniego, L. Wu, L. Noels, D. Tjah- ${ }_{831}^{830}$ janto, E. Casoni, G. Houzeaux, A. Makradi, J. M. Molina- ${ }_{832}$ Aldareguia, M. Vázquez, A. Jérusalem, An XFEM/CZM Im- ${ }_{833}$ plementation for Massively Parallel Simulations of Compos- ${ }_{834}$ ites Fracture, Composite Structures 125 (2015) 542-557. doi : ${ }_{835}$ 10.1016/J . COMPSTRUCT . 2015.01.053.

12] J. Spahn, H. Andrä, M. Kabel, R. Müller, A Multiscale Ap- ${ }_{837}^{836}$ proach for Modeling Progressive Damage of Composite Ma- ${ }_{838}$ terials using Fast Fourier Transforms, Computer Methods s $_{839}$ in Applied Mechanics and Engineering 268 (2014) 871-883. ${ }_{840}$ doi:10.1016/J. CMA. 2013.10.017.

[13] T. T. Nguyen, J. Réthoré, J. Yvonnet, M. C. Baietto, Multi- ${ }_{842}^{84}$ Phase-Field Modeling of Anisotropic Crack Propagation for $_{843}$ Polycrystalline Materials, Computational Mechanics 60 (2) ${ }_{844}$ (2017) 289-314. doi:10.1007/s00466-017-1409-0.

[14] T. Nguyen, J. Yvonnet, Q.-Z. Zhu, M. Bornert, C. Chateau ${ }^{845}$ A Phase-Field Method for Computational Modeling of Inter- ${ }_{847}$ facial Damage Interacting with Crack Propagation in Realis- ${ }_{848}$ tic Microstructures Obtained by Microtomography, Computer ${ }_{849}$ Methods in Applied Mechanics and Engineering $312(2016)_{850}^{849}$ 567-595. doi:10.1016/J. CMA.2015.10.007.

[15] J. Clayton, J. Knap, Phase Field Modeling of Directional Frac- ${ }_{852}^{851}$ ture in Anisotropic Polycrystals, Computational Materials Sci- ${ }_{853}$ ence 98 (2015) 158-169. doi:10.1016/J.COMMATSCI. 2014.11.853 009.
[16] G. Francfort, J.-J. Marigo, Revisiting Brittle Fracture As an Energy Minimization Problem, Journal of the Mechanics and Physics of Solids 46 (8) (1998) 1319-1342. doi: 10.1016/S0022-5096 (98) 00034-9.

[17] L. Wu, L. Noels, L. Adam, I. Doghri, A Multiscale Mean-Field Homogenization Method for Fiber-Reinforced Composites with Gradient-Enhanced Damage Models, Computer Methods in Applied Mechanics and Engineering 233-236 (2012) 164179. doi:10.1016/J.CMA.2012.04.011.

18] V. Kouznetsova, Computational Homogenization for the Multi-Scale Analysis of Multi-Phase Materials, 2002. doi: 10.6100/IR560009.

[19] M. Silani, S. Ziaei-Rad, H. Talebi, T. Rabczuk, A SemiConcurrent Multiscale Approach for Modeling Damage in Nanocomposites, Theoretical and Applied Fracture Mechanics 74 (2014) 30-38. doi:10.1016/j.tafmec.2014.06.009.

[20] I. Benedetti, M. Aliabadi, Multiscale Modeling of Polycrystalline Materials: A Boundary Element Approach to Material Degradation and Fracture, Computer Methods in Applied Mechanics and Engineering 289 (2015) 429-453. doi: 10.1016/J. CMA . 2015.02.018.

[21] F. Feyel, J.-L. Chaboche, FE^2 Multiscale Approach for Modelling the Elastoviscoplastic Behaviour of Long Fibre Sic/Ti Composite Materials, Computer Methods in Applied Mechanics and Engineering 183 (3-4) (2000) 309-330. doi:10.1016/ S0045-7825 (99) 00224-8.

[22] R. E. Miller, E. B. Tadmor, A Unified Framework and Performance Benchmark of Fourteen Multiscale Atomistic/Continuum Coupling Methods, Modelling and Simulation in Materials Science and Engineering 17 (5) (2009) 053001. doi: 10.1088/0965-0393/17/5/053001.

[23] H. Talebi, M. Silani, S. P. Bordas, P. Kerfriden, T. Rabczuk, A Computational Library for Multiscale Modeling of Material Failure, Computational Mechanics 53 (5) (2014) 1047-1071. doi:10.1007/s00466-013-0948-2.

[24] A. Shterenlikht, L. Margetts, Three-Dimensional Cellular Automata Modelling of Cleavage Propagation Across Crystal Boundaries in Polycrystalline Microstructures, Proceedings of the Royal Society of London A: Mathematical, Physical and Engineering Sciences 471 (2177). doi:10.1098/rspa.2015. 0039.

[25] G. Guillemot, C.-A. Gandin, M. Bellet, Interaction Between Single Grain Solidification and Macrosegregation: Application of a Cellular AutomatonFinite Element Model, Journal of Crystal Growth 303 (1) (2007) 58-68. doi:10.1016/J. JCRYSGRO . 2006.12.076.

[26] C. Zheng, D. Raabe, Interaction Between Recrystallization and Phase Transformation During Intercritical Annealing in a Cold-Rolled Dual-Phase Steel: A Cellular Automaton Model, Acta Materialia 61 (14) (2013) 5504-5517. doi:10.1016/J. ACTAMAT . 2013.05.040.

[27] L. Saucedo-Mora, T. James Marrow, Method for the Explicit Insertion of Microstructure in Cellular Automata Finite Element (CAFE) Models Based on an Irregular Tetrahedral Finite Element Mesh: Application in a Multi-Scale Finite Element Microstructure Meshfree Framework (FEMME), Finite Elements in Analysis and Design 105 (2015) 56-62. doi:10.1016/j.finel.2015.07.001.

[28] L. Saucedo-Mora, T. J. Marrow, FEMME: A Multi-Scale Finite Element Microstructure Meshfree Fracture Model for Quasi-Brittle Materials with Complex Microstructures, Engineering Fracture Mechanics 147 (2015) 355-372. doi:10.1016/ j.engfracmech. 2015.05.059.

[29] V. Kouznetsova, M. G. D. Geers, W. A. M. Brekelmans, Multi-Scale Constitutive Modelling of Heterogeneous Materials with a Gradient-Enhanced Computational Homogenization Scheme, International Journal for Numerical Methods in Engineering 54 (8) (2002) 1235-1260. doi:10.1002/nme.541.

[30] V. P. Nguyen, O. Lloberas-Valls, M. Stroeven, L. J. Sluys, Homogenization-Based Multiscale Crack Modelling: From Micro-Diffusive Damage to Macro-Cracks, Computer Meth- 
ods in Applied Mechanics and Engineering 200 (9-12) (2011)926 1220-1236. doi:10.1016/J.CMA.2010.10.013.

[31] L. Shilkrot, R. E. Miller, W. A. Curtin, Multiscale Plasticityg28 Modeling: Coupled Atomistics and Discrete Dislocation Me-929 chanics, Journal of the Mechanics and Physics of Solids 52 (4)930 (2004) 755-787. doi:10.1016/J.JMPS.2003.09.023.

2] M. Silani, H. Talebi, D. Arnold, S. Ziaei-Rad, T. Rabczuk, On932 the Coupling of a Commercial Finite Element Package with933 Lammps for Multiscale Modeling of Materials (jan 2012).

33] H Talebi, M Silani, S P A Bordas, P Kerfriden, T. Rabczuk, Molecular DYNAMICS/XFEM Coupling By A Three-Dimensional Extended Bridging Domain With Applications To Dynamic Brittle Fracture, International Journal for Multiscale Computational Engineering 11 (6) (2013) 527-541. doi: 10.1615/Int JMultCompEng. 2013005838.

34] A. Shterenlikht, L. Margetts, L. Cebamanos, Modelling Fracture in Heterogeneous Materials on HPC Systems using a Hybrid MPI/Fortran Coarray Multi-Scale CAFE Framework, Advances in Engineering Softwaredoi:10.1016/j.advengsoft. 2018.05.008.

[35] I. M. Smith, L. Margetts, The Convergence Variability of Parallel Iterative Solvers, Engineering Computations 23 (2) (2006) 154-165. doi:10.1108/02644400610644522.

[36] M. A. Heroux, D. W. Doerfler, P. S. Crozier, J. M. Willenbring, H. C. Edwards, A. Williams, M. Rajan, E. R. Keiter, H. K. Thornquist, R. W. Numrich, Improving Performance Via Mini-Applications, Tech. Rep. SAND2009-5574, Sandia National Laboratories (2009).

[37] L. Evans, L. Margetts, V. Casalegno, L. Lever, J. Bushell, T. Lowe, A. Wallwork, P. Young, A. Lindemann, M. Schmidt, P. Mummery, Transient Thermal Finite Element Analysis of Cfc-Cu Iter Monoblock using X-Ray Tomography Data, Fusion Engineering and Design 100 (2015) 100-111. doi:10.1016/j. fusengdes.2015.04.048.

38 J. D. Arregui-Mena, L. Margetts, D. V. Griffiths, L. Lever, G. Hall, P. M. Mummery, Spatial Variability in the Coefficient of Thermal Expansion Induces Pre-Service Stresses in Computer Models of Virgin Gilsocarbon Bricks, Journal of Nuclear Materials 465 (2015) 793-804. doi:10.1016/j.jnucmat. 2015. 05.058.

[39] F. Levrero-Florencio, L. Margetts, E. Sales, S. Xie, K. Manda, P. Pankaj, Evaluating the Macroscopic Yield Behaviour of Trabecular Bone using a Nonlinear Homogenisation Approach, Journal of the Mechanical Behavior of Biomedical Materials 61 (2016) 384-396. doi:10.1016/j.jmbbm.2016.04.008.

[40] I. M. Smith, L. Margetts, Portable Parallel Processing for NonLinear Problems, in: VII International Conference on Computational Plasticity, Barcelona, 2003.

[41] L. Evans, L. Margetts, P. Lee, C. Butler, E. Surrey, Image Based in Silico Characterisation of the Effective Thermal Properties of a Graphite Foam, Carbon 143 (2019) 542-558. doi:10.1016/J.CARBON. 2018.10.031.

[42] S. Hewitt, L. Margetts, A. Revell, Parallel Performance of an Open Source Fluid Structure Interaction Application, in: Proceedings of the 25th UKACM Conference on Computational Mechanics, Birmingham, 2017.

[43] N. M. Newmark, A Method of Computation for Structural Dynamics, Journal of the engineering mechanics division 85 (3) (1959) $67-94$.

[44] I. M. Smith, D. V. Griffiths, L. Margetts, Programming the Finite Element Method, 5th Edition, John Wiley \& Sons, Ltd, 2014.

[45] S. Li, S. Ghosh, Extended Voronoi Cell Finite Element Model for Multiple Cohesive Crack Propagation in Brittle Materials, International Journal for Numerical Methods in Engineering 65 (7) (2006) 1028-1067. doi:10.1002/nme.1472.

46] J. E. Bishop, Simulating the Pervasive Fracture of Materials and Structures using Randomly Close Packed Voronoi Tessellations, Computational Mechanics 44 (4) (2009) 455-471. doi: 10.1007/s00466-009-0383-6.

[47] J. Gilman, Cleavage, Ductility and Fracture in Crystals, in:
Fracture, 1959

[48] M. Geers, V. Kouznetsova, W. Brekelmans, Multi-Scale Computational Homogenization: Trends and Challenges, Journal of Computational and Applied Mathematics 234 (7) (2010) 2175-2182. doi:10.1016/j.cam.2009.08.077.

[49] M. F. Horstemeyer, Multiscale Modeling: A Review, in: Practical Aspects of Computational Chemistry, Springer Netherlands, Dordrecht, 2009, pp. 87-135. doi:10.1007/ 978-90-481-2687-3_4.

[50] ARCHER: The Latest UK National Supercomputing Service. 